\title{
A new bidirectional associative fuzzy cognitive dynamical system
}

\author{
K.Thirusangu ${ }^{1^{\star}}$, P. Elumalai ${ }^{2}$ and A. Praveenprakash ${ }^{3}$ \\ ${ }^{1}$ Department of Mathematics, SIVET College, Gowrivakkam, Chennai 600 073, India \\ ${ }^{2}$ Department of Mathematics, Government Arts College, Thiruvannamalai-606603, TamilNadu, India \\ ${ }^{3}$ Department of Mathematics, Hindustan University, Chennai 603 103, India \\ 1*kthirusangu@gmail.com
}

\begin{abstract}
In this paper, we introduce a new Fuzzy bimodel called Bidirectional Associative Fuzzy Cognitive Maps (BAFCM). We state the advantages of this system and its application in addressing to socio-economic problems.
\end{abstract}

Keywords: FCM, BAM, BAFCM, fixed point, limit cycle, bihidden pattern.

Introduction
$\quad$ Neural networks and fuzzy systems estimate inputoutput functions. Both are trainable dynamical systems. Fuzzy models are mathematical tools. They have been introduced by L.A. Zadeh (1965) to study and analyze political decisions, neural networks, social problems etc., (Klir et al., 1998). Among the various fuzzy models, some of the important models which have been used to study these problems are Fuzzy Associative Memories (FAM), Fuzzy Cognitive Maps (FCM) and Fuzzy Relational Maps (FRM), Fuzzy Cognitive Relational Maps (FCRM) and $\mathrm{Bi}$ Associative Memories (BAM). The BAM model was introduced by Bart Kosko (Kosko, 1988; 2001). Yet already engineers have successfully applied fuzzy systems in many commercial areas. It is used to model several types of problems varying from gastric appetite behavior, popular political developments etc. It is also used to model in robotics like plant control. This model works on the opinion of experts.

These models have been used to study various social problems. In paricular, the problem of health hazards caused by chemical pollutions, the causes for school dropouts which ultimately lead to child labour have been studied in the literature (Balasangu et al., 2007, 2009, 2011; Praveen prakash, 2010). In order to bring out much stronger relationship among the attributes, a new model called Induced Bi-directional Associative Memories (IBAM) (Pathinathan et.al 2005).

In this paper we introduce a new fuzzy bimodel called Bidirectional Associative Fuzzy Cognitive Maps (BAFCM). This new bimodel can act simultaneously as a Bidirectional Associative Memories (BAM) as well as Fuzzy Cognitive Maps (FCMs). This model is best suited when the study under consideration is one with an unsupervised data. This model can give a bihidden pattern. We have mentioned the advantages and merits of this bimodel. As an application the health hazards of rag pickers has been discussed. Based on our observation, we provide some suggestions to overcome this problem.

\section{What is the need for this model?}

Suppose we have a problem at hand, and the data related with it happens to be an unsupervised one, obtained from survey as well as interviews and discussions, it may so happen that the attributes in one case or under one expert may not be a disjoint one and under another expert the attributes may be disjoint. In such a case, if we want to consider a pair of attributes one relates with the BAM dynamical system and the other with a FCM dynamical system and if we wish to see the step-by-step or stage-by-stage effect of the attributes on the system, we need a dynamical system. Hence we introduce a new dynamical bisystem which is capable of analyzing the problem at hand and giving the effects of the attributes on the dynamical bisystem.

Further, as all socio economic problems cannot be given solution in terms of statistical data or be exhibited by numerical values, we feel only this model is capable of analyzing the problem and will give the effects of the attributes. The uniqueness of this model is that it can give a pair of hidden patterns i.e. a different pairs of sets of resultants. By this one can understand or study or get to know the underlying effect of a pair of attributes over a dynamical bisystem.

Bidirectional associative fuzzy cognitive maps (BAFCM) bimodel

We first give the notion of Bidirectional Associative Memories and Fuzzy Cognitive Maps and their functioning.

Description of the BAM model

A group of neurons forms a field. Neural networks contain many fields of neurons. Suppose $F_{x^{-}}$denotes a neuron field which contains $n$-neurons and $F_{y}$ - denotes a neuron field which contains $p$ - neurons. The neuronal dynamical system is described by a system of first order differential equations that govern the time evolution of the neuronal activation

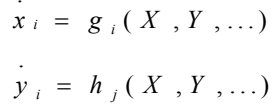

where $x_{i}$ and $y_{j}$ denote, respectively, the activation time function of the $i^{\text {th }}$ neuron in $\mathrm{F}_{\mathrm{x}}$ and the $j^{\text {th }}$ neuron in $\mathrm{F}_{\mathrm{y}}$. The over dot denotes time differentiation, $g_{i}$ and $h_{j}$ are some functions of $X$ and $Y$ etc., where 
$X(t)=\left(x_{1}(t), \ldots, x_{n}(t)\right)$

$Y(t)=\left(y_{1}(t), \ldots, y_{p}(t)\right)$

define the state of the neuronal dynamical system at time t.

Let us suppose that the field $F_{x}$ with $n$-neurons is synoptically connected to the field $F_{y}$ with p-neurons. Let $\left(m_{i j}\right)$ be a synapse (junction) from the $i^{\text {th }}$ neuron in $F_{x}$ and the $j^{\text {th }}$ neuron in $\mathrm{F}_{\mathrm{y}}, \mathrm{m}_{\mathrm{ij}}$ can be positive, negative or zero $(1 \leq i \leq n$ and $1 \leq j \leq p)$. The synaptic matrix $\mathrm{M}$ is an $\mathrm{n} \times \mathrm{p}$ matrix of real numbers whose entries are synaptic efficacies $m_{i j}$. The matrix $M$ or the network describes the forward projection from neuronal field $F_{x}$ to the neuronal field $F_{y}$. Similarly, a $p \times n$ synaptic matrix $N$ or the network describes the backward projection from $F_{y}$ to $F_{x}$. The network is said to be bi-directional network if $M=N^{\top}$ and $N=M^{\top}$. When the activation dynamics of the neuronal fields $F_{x}$ and $F_{y}$ lead to the overall stable behaviour, the bi-directional networks are called as $\mathrm{Bi}$ directional Associative Memories or BAM.

An additive model is defined by a system of $n+p$ coupled first-order differential equations that interconnects the fields $F_{x}$ and $F_{y}$ through the constant synaptic matrices $\mathrm{M}$ and $\mathrm{N}$ described earlier.

$\dot{x}_{i}=-A_{i} x_{i}+\sum_{j=1}^{p} S_{j}\left(y_{j}\right) n_{j i}+I_{i}$

$\dot{y}_{i}=-A_{j} y_{j}+\sum_{i=1}^{p} S_{i}\left(x_{i}\right) m_{j i}+J_{j}$

$S_{i}\left(x_{i}\right)$ and $S_{j}\left(y_{j}\right)$ Denote, respectively, the signal function of the the neuron in the field $F_{x}$ and the signal function of the $j^{\text {th }}$ neuron in the field $F_{y}$. Discrete additive activation models correspond to neuron with threshold signal functions. The neurons can assume only two values, ON and OFF, where On represents the signal vaule +1 and OFF represents 0 or -1 ( -1 when the representation is bipolar).

At each moment, each neuron can randomly decide whether to change state, or whether to emit a new signal given its current activation. The BAM is a non -adaptive additive bivalent neural network.

In real life problems the entries of the constant synaptic matrix $M$ depend upon the experts' opinion or the feelings of the investigator. The synaptic matrix is given a weightage according to the experts' opinion or their feelings.

If $x \in F_{x}$ and $y \in F_{y}$, the forward projection from $\mathrm{F}_{\mathrm{x}}$ to $\mathrm{F}_{\mathrm{y}}$ is defined by the matrix $\mathrm{M}$ as

$\left\{F\left(x_{i}, y_{j}\right)\right\}=\left(m_{i j}\right)=M, 1 \leq i \leq n, 1 \leq j \leq p$.

The backward projections are defined by the matrix $M^{\top}$ as

$$
\left\{F\left(y_{j}, x_{i}\right)\right\}=\left(m_{j i}\right)=M^{T}, 1 \leq i \leq n, 1 \leq j \leq p .
$$

A BAM system $\left(F_{x}, F_{y}, M\right)$ is bi-directionally stable if all inputs converge to fixed -point equilbria. The change of the state of the system leads to a fixed point or a limit cycle. The bi-directional stability is a dynamic equilibrium. The same signal information flows back and forth in a bidirectional fixed point.

Let us suppose that $A$ denotes a binary $n$-vector and $B$ denotes a binary $p$-vectors. Let $A$ be the initial input to the BAM system. Then the BAM equilibrates to a bidirectional fixed point $\left(A_{f}, B_{f}\right)$ as

$$
\begin{gathered}
A^{\prime} \leftarrow M^{T} \leftarrow B \\
A^{\prime} \rightarrow M^{\prime} \rightarrow B^{\prime} \\
A^{\prime \prime} \leftarrow M^{T} \leftarrow B^{\prime} \\
\cdot \\
A_{f} \rightarrow M^{\prime} \rightarrow B_{f}
\end{gathered}
$$

where $\mathrm{A}^{\prime}, \mathrm{A}^{\prime \prime}, \ldots$ and $\mathrm{B}^{\prime}, \mathrm{B}^{\prime \prime}, \ldots$ represent intermediate or transient signal state vectors between $A$ and $A_{f}$ and $B$ and $B_{f}$ respectively. The fixed point of a bi-directional system is time dependent. The fixed point for the initial input vectors can be attained at different times. Based on the synaptic matrix $M$ which is developed by the expert's opinion or their feelings, the time at which bi-directional stability is attained also varies accordingly.

Fuzzy Cognitive Maps

Fuzzy Cognitive Maps can be described by a directed graph. The concepts are taken as nodes. If there is a relationship between the attributes, we draw an edge between them in the following way. The directed edge $e_{i j}$ from casual concept $C_{i}$ to concept $C_{j}$ measures how much $C_{i}$ causes $C_{j}$. The time varying concept function $C_{i}(t)$ measures the non-negative occurrence of some fuzzy event, perhaps the strength of a political sentiment, historical trend or Military objective. If increase (or decrease) in one concept leads to increase (or decrease) in another, then we give the value 1 . If there exists no relation between two concepts the value 0 is given. If increase (or decrease) in one concept decreases (or increases) another, then we give the value -1 .

Definition 3.1 When the nodes of the FCM are fuzzy sets then they are called as fuzzy nodes.

The FCMs with edge weights or casualities from the set $\{-1,0,1\}$, are called simple FCMs.

Definition 3.2 Consider the nodes/ concepts $\mathrm{C}_{1}, \mathrm{C}_{2}$, ......., $\mathrm{C}_{\mathrm{n}}$ of the FCM. Suppose the directed graph is drawn using edge weight $e_{i j} \in\{0,1,-1\}$. The matrix $M$ be defined by $M=\left(e_{i j}\right)$ where $e_{i j}$ is the weight of the directed edge $C_{i} C_{j}$. M is called the adjacency matrix of the FCM, also known as the connection matrix of the FCM. It is important to note that all matrices associated with an FCM are always square matrices with diagonal entries as zero.

Let $C_{1}, C_{2}, \ldots \ldots, C_{n}$ be the nodes of an FCM. $A=\left(a_{1}, a_{2}\right.$, $\left.\ldots . a_{n}\right)$ where $a_{i} \in\{0,1\}$. 
The above vector $A$ is called the instantaneous state vector and it denotes the ON-OFF position of the node at an instant.

$a_{i}=\left\{\begin{array}{l}0 \text { if } a_{i} \text { is OFF } \\ 1 \text { if } a_{i} \text { is ON , for } i=1,2, \ldots, n .\end{array}\right.$

Definition 3.3 Let $\mathrm{C}_{1}, \mathrm{C}_{2}, \ldots, \mathrm{C}_{\mathrm{n}}$ be the nodes of an FCM. Let $\overline{C_{1} C_{2}}, \overline{C_{2} C_{3}}, \overline{C_{3} C_{4}}, \ldots ., \overline{C_{i} C_{j}}$ be the edges of the $\operatorname{FCM}(i \neq j)$. Then the edges form a directed cycle. An

FCM is said to be cyclic if it possesses a directed cycle. An FCM is said to be acyclic if it does not possess any directed cycle. An FCM with cycles is said to have a feedback. When there is a feedback in an FCM, i.e., when the casual relations flow through a cycle in a revolutionary way, the FCM is called a dynamical system. Let $\mathrm{C}_{1} \mathrm{C}_{2}, \mathrm{C}_{2} \mathrm{C}_{3}, \ldots, \mathrm{C}_{\mathrm{n}-1} \mathrm{C}_{\mathrm{n}}$ be a cycle. When $\mathrm{C}_{\mathrm{i}}$ is switched on and if the causality flows through the edges of a cycle and if it again causes $C_{i}$, we say that the dynamical system goes round and round. This is true for any node $C_{i}$, for $i=1,2, \ldots, n$. The equilibrium state for this dynamical system is called the hidden pattern. If the equilibrium state of a dynamical system is a unique state vector, then it is called a fixed point. If the FCM settles down with a state vector repeating in the form $A_{1} \rightarrow A_{2} \rightarrow A_{3} \rightarrow A_{4} \rightarrow$ $\ldots \ldots . . \rightarrow A_{1}$, then this equilibrium is called a limit cycle.

Example 3.4 Consider an FCM with $\mathrm{C}_{1}, \mathrm{C}_{2}, \ldots \ldots, \mathrm{C}_{\mathrm{n}}$ as nodes. For example let us start the dynamical system by switching on $\mathrm{C}_{1}$. Let us assume that the FCM settles down with $C_{1}$ and $C_{n}$ as $O N$. That is, the state vector remains as $(1,0,0, \ldots ., 0,1)$.

This state vector $(1,0,0, \ldots \ldots, 0,1)$ is called the fixed point.

Notation: Suppose $A=\left(a_{1}, a_{2}, \ldots \ldots \ldots, a_{n}\right)$ is a vector which is passed into a dynamical system $M$. Then $A M=($ $\left.a^{\prime}{ }_{1}, a^{\prime}{ }_{2} \ldots \ldots \ldots . . . . a_{n}\right)$. After thresholding and updating the vector suppose we get $\left(b_{1}, b_{2}, \ldots \ldots \ldots, b_{n}\right)$, then we denote this vector by

$\left(a_{1}^{\prime}, a_{2}^{\prime} \ldots \ldots \ldots . . . a_{n}\right) \quad \longrightarrow \quad\left(b_{1}, b_{2}, \ldots \ldots \ldots, b_{n}\right)$

Thus the symbol, $\longrightarrow$ means the resultant vector has been thresholded and updated.

The FCM model works as follows: We pass state vectors I repeatedly through the FCM connection matrix $M$, thresholding or non linearly transforming the result after each pass. Independent of the FCMs size, it quickly settles down to a temporal associative memory limit cycle or fixed point which is the hidden pattern of the system for that state vector I. The limit cycle or fixed-point inference summarizes the joint effects of all the interacting fuzzy knowledge.

\section{The new BAFCM bimodel}

In this section we describe the new bimodel, the advantages of this model and its merits.
Vol. 5 No. 3 (Mar 2012)

ISSN: 0974- 6846

Definition 3.5: Let $S=S_{1} \cup S_{2}$, where $S_{1}$ and $S_{2}$ are nonempty sets; with $S_{1} \nsubseteq \pm S_{2}$ and $\quad S_{2} \not \subset S_{1}$ then we call $\mathrm{S}$ as a biset.

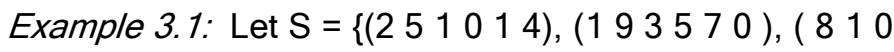

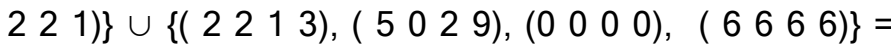
$\mathrm{S}_{1} \cup \mathrm{S}_{2}$, clearly $\mathrm{S}$ is a biset.

Definition 3.6: Let $\mathrm{V}_{1}=\left(\mathrm{v}_{1}, \mathrm{v}_{2}, \ldots, \mathrm{v}_{\mathrm{n}}\right)$ and $\mathrm{V}_{2}=\left(\mathrm{v}_{1}, \mathrm{v}_{2}\right.$, $\left.\mathrm{v}_{3}, \ldots, \mathrm{v}_{\mathrm{m}}\right)$ be two vectors of length $\mathrm{n}$ and $\mathrm{m}$ respectively.

Then $\mathrm{V}=\mathrm{V}_{1} \cup \mathrm{V}_{2}$ is a bivector.

Example 3.2: Let $\mathrm{V}=\mathrm{V}_{1} \cup \mathrm{V}_{2}=\left(\begin{array}{l}8 \\ 8\end{array} 4193\right) \cup\left(\begin{array}{lll}3 & 4 & 5\end{array}\right.$ ), $V$ is a bivector. If $V=V_{1} \cup V_{2}=(000000) \cup(0000)$, then

$\mathrm{V}$ is a zero bivector. If $\mathrm{V}=\mathrm{V}_{1} \cup \mathrm{V}_{2}=(111111) \cup(1111)$ then $\mathrm{V}$ is a unit bivector.

Definition 3.7: A matrix $E$ is said to be a bimatrix if $E=$ $E_{1} \cup E_{2}$ where $E_{1}$ and $E_{2}$ are two different matrices.

Example 3.3: A matrix $E$ of the form $E=E_{1} \cup E_{2}=\left(\begin{array}{l}375 \\ \hline\end{array}\right.$

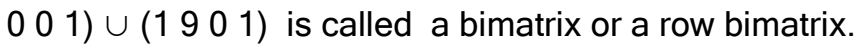
Example 3.4:

Let $\mathrm{E}=\mathrm{E}_{1} \cup \mathrm{E}_{2}=$

$\left[\begin{array}{lllll}6 & 5 & 0 & 0 & 2 \\ 1 & 1 & 4 & 0 & 1 \\ 7 & 0 & 1 & 2 & 6 \\ 5 & 2 & 0 & 8 & 3 \\ 2 & 4 & 1 & 7 & 0\end{array}\right] \cup\left[\begin{array}{lllll}2 & 3 & 2 & 1 & 9 \\ 1 & 4 & 9 & 4 & 0 \\ 8 & 6 & 5 & 0 & 6 \\ 1 & 6 & 9 & 8 & 1 \\ 0 & 2 & 5 & 1 & 3\end{array}\right]$

$E$ is a square bimatrix.

Definition 3.8: Let $G=G_{1} \cup G_{2}$ where $G_{1}$ and $G_{2}$ are two distinct graphs then we call $G$ as a bigraph.

Definition 3.9: Let $E=E_{1} \cup E_{2}$ be a bimatrix where $E_{1}$ is a $\mathrm{m} \times \mathrm{n}$ matrix and $\mathrm{E}_{2}$ is a $\mathrm{p} \times \mathrm{s}$

matrix. If $V=V_{1} \cup V_{2}$ is a bivector such that $V_{1}$ has $m$ components and $V_{2}$ has $p$ components then the product of $V$ with $E$ is defined as $V E=\left(V_{1} \cup V_{2}\right)\left(E_{1} \cup E_{2}\right)=V_{1} E_{1} \cup$ $V_{2} E_{2}$ where $V_{1} E_{1}$ is a $1 \times n$ matrix and $V_{2} E_{2}$ is a $1 \times S$ matrix or more mathematically; $V_{1} E_{1} \cup V_{2} E_{2}=A_{1} \cup A_{2}$ is a bivector or a row bivector.

Definition 3.10: Let $E=E_{1} \cup E_{2}$ be a bimatrix. Then the bitranspose of the bimatrix $E$ is defined as $E^{t}=\left(E_{1} \cup E_{2}\right)^{t}$ $=\mathrm{E}_{1}{ }^{\mathrm{t}} \cup \mathrm{E}_{2}{ }^{\mathrm{t}}$.

Definition 3.11: A Bidirectional Associative Fuzzy Cognitive Maps (BAFCM) is a directed special bigraph with concepts like policies, events, etc as nodes and causalities as edges. It represents causal relationship between concepts. In a BAFCM we call the pair of associated nodes as binodes. If the order of the bimatrix associated with BAFCM is a $p \times m$ matrix and $a n \times n$ square matrix then the binodes are bivectors of length $(p, n)$ or length $(m, n)$.
Research article

CIndian Society for Education and Environment (iSee)
"Fuzzy bimodel"

http://www.indjst.org
Thirusangu et al. Indian J.Sci.Technol. 
Let $V=V_{1} \cup V_{2}=\left(v_{1}{ }^{1}, v_{2}{ }^{1}, v_{3}{ }^{1}, \ldots, v_{m}{ }^{1}\right) \cup\left(v_{1}{ }^{2}, v_{2}{ }^{2}, v_{3}{ }^{2} . ., v_{m}{ }^{2}\right)$ where $v_{i} \in\{0,1\} ; V$ is called the instantaneous state bivector and it denotes the ON-OFF position of the binode at an instant. $v_{j}^{i}=0$ if $v_{j}^{i}$ is OFF, $v_{j}^{i}=1$ if $v_{j}^{i}$ is ON, for $1 \leq i$ $\leq 2$ and $1 \leq \mathrm{j} \leq \mathrm{m}, \mathrm{n}$.

Definition 3.12: Consider the binodes biconcepts $\left\{X_{1} \ldots X_{p}\right\}$ and $\left.\left\{Y_{1} \ldots . Y_{m}\right\}\right\}$ of the BAM and $\left\{C_{1}{ }^{1} C_{2}{ }^{1} \ldots . . C_{n}{ }^{1}\right\}$ of the FCM of the BAFCM bimodel. Suppose the directed graph is drawn using the edge biweight $\mathrm{e}_{\mathrm{ij}}{ }^{1}=\{0, \pm 1, \pm 2$, $\pm 3, \pm 4, \pm 5\} ; e_{i j}{ }^{2}=\{0, \pm 1\}$. The bimatrix $E=E_{1} \cup E_{2}$ is defined by $\mathrm{e}_{\mathrm{ks}}{ }^{1} \cup \mathrm{e}_{\mathrm{ij}}{ }^{2}$ where $\mathrm{e}_{\mathrm{ks}}{ }^{1}$ is the directed edge of $X_{k} Y_{s}$ and $e_{i j}^{2}$ is the weight of the directed edge $C_{i} C_{j} . E=$

$E_{1} \cup E_{2}$ is called adjacency bimatrix of the new BAFCM bimodel, also known as the connecting relational bimatrix of the new BAFCM bimodel.

Definition 3.13: The new BAFCMs with edge biweight $\{1,0,-1\}$ are called simple BAFCMs. Let $\left\{\left(X_{1}, \ldots X_{m}\right),\left(Y_{1} \ldots Y_{n}\right)\right\} \cup\left\{C_{1}, \ldots C_{p}\right\}$ be the binodes of an BAFCM. $V=V_{1} \cup V_{2}=\left(x_{1}, \ldots x_{m}\right)\left(\operatorname{or}\left(y_{1}, \ldots y_{n}\right)\right) \cup\left(z_{1}, \ldots z_{p}\right)$ where $\mathrm{x}_{\mathrm{i}}, \mathrm{y}_{\mathrm{j}}, \mathrm{z}_{\mathrm{k}} \in\{0,1\} ; 1 \leq \mathrm{i} \leq \mathrm{m}, 1 \leq \mathrm{j} \leq \mathrm{n}$ and $1 \leq \mathrm{k} \leq \mathrm{p}$. V is called instantaneous state bivector and it denotes the ON-OFF position of the node at an instant.

$x_{j}=0$ if $x_{j}$ is OFF and $x_{j}=1$ if $x_{j}$ is ON for $1 \leq j \leq m$

$y_{i}=0$ if $y_{i}$ is OFF and $y_{i}=1$ if $y_{i}$ is ON for $1 \leq i \leq n$

$z_{k}=0$ if $z_{k}$ is OFF and $z_{k}=1$ if $z_{k}$ is ON for $1 \leq k \leq p$ $\mathrm{i}=1,2,3 \ldots \mathrm{m}$

Definition 3.14: Let $\left\{\left(X_{1}, \ldots X_{m}\right),\left(Y_{1} \ldots . Y_{n}\right)\right\} \cup\left\{C_{1}, \ldots C_{p}\right\}$ be the binodes of an BAFCM.. Let $X_{k} Y_{s} \cup C_{i} C_{j}$ be the biedges of the BAFCMs; $1 \leq \mathrm{k} \leq \mathrm{m}, 1 \leq \mathrm{s} \leq \mathrm{n}, 1 \leq \mathrm{i}, \mathrm{j} \leq \mathrm{p},(\mathrm{i} \neq \mathrm{j})$.

Then the biedges form a directed bicycle. An BAFCM is said to be bicyclic if it possesses a directed bicycle. An BAFCM is said to be abicyclic if it does not possess any directed bicycle.

Definition 3. 15: An BAFCM with bicycles is said to have a feedback. When there is a feed back in an BAFCM, i.e., when the casual relations flow through a cycle in a revolutionary way, the BAFCM is called a dynamical bisystem.

Definition 3. 16: Let $\left\{\left(X_{i} Y_{j}\right)\left(\right.\right.$ or $\left.\left.Y_{j} X_{i}\right) \mid 1 \leq \mathrm{i} \leq \mathrm{m}, 1 \leq \mathrm{j} \leq \mathrm{n}\right\} \cup$ $\left\{\mathrm{C}_{1} \mathrm{C}_{2}, \mathrm{C}_{2} \mathrm{C}_{3} \ldots \ldots . . . \mathrm{C}_{\mathrm{n}-1} \mathrm{C}_{n}\right\}$ be a bicycle. If $\mathrm{Y}_{\mathrm{j}}\left(\right.$ or $\mathrm{X}_{\mathrm{i}}$ ) is switched $\mathrm{ON}$ and if the casuality flows through the edges

of the bicycle and if it again causes $Y_{j}\left(\right.$ or $\left.X_{i}\right) \cup C_{k}$ we say that the dynamical bisystem goes round and round. This

is true for the binodes $Y_{j}\left(\right.$ or $\left.X_{i}\right) \cup C_{k}$ for $1 \leq \mathrm{j} \leq \mathrm{n}$ (or $1 \leq \mathrm{i}$ $\leq \mathrm{m}), \quad 1 \leq \mathrm{k} \leq \mathrm{n}$. The equilibrium bistate for the dynamical bisystem is called the hidden bipattern. If the equilibrium bistate of the dynamical bisystem is a unique bistate bivector then it is called fixed bipoint.

Example 3.5: Consider the BAFCMs with $\left(\mathrm{X}_{1}, \ldots \mathrm{X}_{\mathrm{p}}\right)$ (or $\left.Y_{1} \ldots . Y_{m}\right)\left(C_{1}, \ldots . . C_{n}\right)$ as binodes. For instant if we start the dynamical bisystem by switching $Y_{1}$ (or $\left.X_{1}\right) \cup C_{1} O N$. Let us assume the BAFCM settles down with $\left(Y_{1}\right.$ and
$\left.Y_{m}\right) \operatorname{or}\left(X_{1}\right.$ and $\left.\left.X_{p}\right)\right) \cup C_{1}$ and $C_{n}$. That is the state bivector remains as $(100 \ldots 1)$ in $Y($ or $(100 \ldots 1)$ in $X \cup(100 \ldots 01)$. This state bivector is called the fixed bipoint. It is to be noted that in the case of BAFCM we get a pair of fixed bipoint say $A=A_{1} \cup X$ and $A=A_{1} \cup Y ; X$ denote the state vector in the Fx field of the BAM component of the BAFCM and $Y$ denotes the state vector of the Fy field of the BAM component of the BAFCM bimodel.

Definition 3.17: If the BAFCM settles down with a bistate bivector repeating in the form $\quad B_{1} \rightarrow B_{2} \rightarrow \ldots . . B_{j} \rightarrow B_{1}$ (or $D_{1}$ $\left.\rightarrow D_{2} \rightarrow \ldots \rightarrow D_{k} \rightarrow D_{1}\right) \cup A_{1} \rightarrow A_{2} \rightarrow \ldots . . \rightarrow A_{1}$ then this equilibrium is called a limit bicycle.

Definition 3.18: The biedges $\mathrm{e}_{\mathrm{ij}}=\left(\mathrm{e}_{\mathrm{ks}}{ }^{1}\right) \cup\left(\mathrm{e}_{\mathrm{ij}}{ }^{2}\right)$ take the values in fuzzy casual biinterval

$[-1,1] \cup[-1,1]$.

i) $e_{i j}=0$ indicates no causality between the binodes.

ii) $\mathrm{e}_{\mathrm{ij}}>0$ implies that both $\mathrm{e}_{\mathrm{ij}}{ }^{2}>0$ and $\mathrm{e}_{\mathrm{ks}}{ }^{1}>0$; implies increase in the binodes $C_{i} \cup X_{k}\left(\right.$ or $\left.Y_{s}\right)$; implies increase in the binodes $C_{j} \cup Y_{s}\left(\right.$ or $\left.X_{s}\right)$.

iii) $e_{i j}<0$ implies that both $e_{i j}{ }^{2}<0$ and $e_{k s}{ }^{1}<0$; similarly decrease in the binodes $C_{i} \cup X_{k}\left(\right.$ or $\left.Y_{s}\right)$; implies decrease in the binodes $C_{j} \cup Y_{s}\left(\right.$ or $\left.X_{k}\right)$

However, unlike the FCM and BAM model we can have the following possibilities other than that of $e_{i j}=0, e_{i j}>0$ and $\mathrm{e}_{\mathrm{ij}}<0$.

i) $e_{i j}=\left(e_{i j}{ }^{2}\right) \cup\left(e_{k s}{ }^{1}\right)$ can be such that $\left(e_{i j}{ }^{2}\right)=0$ and $\left(e_{k s}{ }^{1}\right)$ $>0$. No relation in one binode and an increase in other node.

ii) $\mathrm{e}_{\mathrm{ij}}=\left(\mathrm{e}_{\mathrm{ij}}{ }^{2}\right) \cup\left(\mathrm{e}_{\mathrm{ks}}{ }^{1}\right)$ we can have $\left(\mathrm{e}_{\mathrm{ij}}{ }^{2}\right)=0$ and $\left(\mathrm{e}_{\mathrm{ks}}{ }^{1}\right)<0$. No causality in the FCM node and decreasing relation in the BAM mode.

iii) $\mathrm{e}_{\mathrm{ij}}=\left(\mathrm{e}_{\mathrm{ij}}{ }^{2}\right) \cup\left(\mathrm{e}_{\mathrm{ks}}{ }^{1}\right)$ we can have $\left(\mathrm{e}_{\mathrm{ij}}{ }^{2}\right)=<0$ and $\left(\mathrm{e}_{\mathrm{ks}}{ }^{1}\right)>0$ iv) In $e_{i j}=\left(e_{i j}{ }^{2}\right) \cup\left(e_{k s}{ }^{1}\right)$ we can have $\left(e_{i j}{ }^{2}\right)<0$ and $\left(e_{k s}{ }^{1}\right)=0$ v) In $e_{i j}=\left(e_{i j}{ }^{2}\right) \cup\left(e_{k s}^{1}\right)$ we can have $\left(e_{i j}{ }^{2}\right)>0$ and $\left(e_{k s}{ }^{1}\right)=0$ vi) In $\mathrm{e}_{\mathrm{ij}}=\left(\mathrm{e}_{\mathrm{ij}}{ }^{2}\right) \cup\left(\mathrm{e}_{\mathrm{ks}}{ }^{1}\right)$ we can have $\left(\mathrm{e}_{\mathrm{ij}}{ }^{2}\right)>0$ and $\left(\mathrm{e}_{\mathrm{ks}}{ }^{1}\right)<$ 0 .

Thus in the case of BAFCM we can have 9 possibilities where as in FCMs or BAMs we have only 3 possibilities. Thus the extra 6 possibilities can help in making the solution of the problem more sensitive or accurate.

\section{How this new bimodel works?}

\section{Algorithm}

Input: The dynamical bisytem $\mathrm{E}=\mathrm{E}_{1} \cup \mathrm{E}_{2}$ and the initial bivector $A=A_{1} \cup A_{2}$.

Step 1: Take the system as a bimatrix $E=(m \times p) \cup(n \times$ n) and

$A=(1 \times m)($ or $(1 \times p)) \cup(1 \times n)$ as the input bivector.

Step 2: Pass the bivector $A=A_{1} \cup A_{2}$ into a dynamical bisystem $E=E_{1}\left(\operatorname{or}\left(E_{1}^{\top}\right)\right) \cup E_{2}$.
Research article

Clndian Society for Education and Environment (iSee)
Thirusangu et al. Indian J.Sci.Technol. 
Let the resultant be $A E=A_{1} E_{1}\left(\operatorname{or}\left(A_{1} E_{1}^{\top}\right)\right) \cup A_{2} E_{2}=\left(y_{1}^{\prime}\right.$, $\left.\mathrm{y}_{2}^{\prime}, \ldots, \mathrm{y}_{\mathrm{p}}^{\prime}\right)\left(\right.$ or $\left(\mathrm{z}_{1}^{\prime}, \mathrm{z}_{2}^{\prime}, \ldots, \mathrm{z}_{\mathrm{m}}\right) \cup\left(\mathrm{x}_{1}^{\prime}, \mathrm{x}_{2}^{\prime}, \ldots, \mathrm{x}_{\mathrm{n}}^{\prime}\right)$.

Step 3: Threshold and update this $\mathrm{AE}$ using the signal function $S$ as:

$\mathrm{S}\left(\mathrm{y}_{\mathrm{j},}\right)=0$ if $\mathrm{y}_{\mathrm{j},} \leq 0 ; \quad 1 \leq \mathrm{j} \leq \mathrm{p}$

$S\left(y_{j}\right)=1$ if $y_{j}>0 ; \quad 1 \leq j \leq p$

$S\left(z_{k}\right)=0$ if $z_{k} \leq 0 ; \quad 1 \leq k \leq m$

$S\left(z_{k}\right)=1$ if $z_{k}>0 ; \quad 1 \leq k \leq m$

$S\left(x_{i}\right)=0$ if $x_{i} \leq 0 ; \quad 1 \leq i \leq n$

$\mathrm{S}\left(\mathrm{x}_{\mathrm{i}}\right)=1$ if $\mathrm{x}_{\mathrm{i}}>0 ; \quad 1 \leq \mathrm{i} \leq \mathrm{n}$

That is if $\left(\mathrm{y}_{1}^{\prime}, \mathrm{y}_{2}^{\prime}, \ldots, \mathrm{y}_{\mathrm{p}}{ }^{\prime}\right)\left(\operatorname{or}\left(\mathrm{z}_{1}^{\prime}, \mathrm{z}_{2}, \ldots, \mathrm{z}_{\mathrm{m}}\right)\right) \cup\left(\mathrm{x}_{1}^{\prime}, \mathrm{x}_{2}^{\prime}, \ldots, \mathrm{x}_{\mathrm{n}}^{\prime}\right)$ $\longrightarrow\left(y_{1}, y_{2}, \ldots, y_{p}\right)$

(or $\left.\left(\mathrm{z}_{1}, \mathrm{z}_{2}, \ldots, \mathrm{z}_{\mathrm{m}}\right)\right) \cup\left(\mathrm{x}_{1}, \mathrm{x}_{2} \ldots \mathrm{x}_{\mathrm{n}}\right)$.

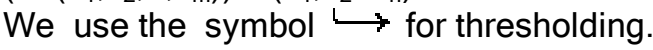

Moreover, keep the state $x_{j}$ as ON if it was in ON position in $\mathrm{A}_{2}$.

Then, the values of $\mathrm{x}_{\mathrm{i}}, \mathrm{y}_{\mathrm{j}}, \mathrm{z}_{\mathrm{k}}$ are either zero or 1 , for $1 \leq \mathrm{i}$ $\leq \mathrm{n} ; 1 \leq \mathrm{j} \leq \mathrm{p}$; and $1 \leq \mathrm{k} \leq \mathrm{m}$.

Step 4: Assign: $\quad \mathrm{B}=\left(\mathrm{B}_{1} \cup \mathrm{B}_{2}\right) \leftarrow\left(\left(\mathrm{y}_{1}, \mathrm{y}_{2}, \ldots, \mathrm{y}_{\mathrm{p}}\right)\right.$ (or

$\left.\left.\left(\mathrm{z}_{1}, \mathrm{z}_{2}, \ldots, \mathrm{z}_{\mathrm{m}}\right)\right) \cup\left(\mathrm{x}_{1}, \mathrm{x}_{2} \ldots \mathrm{x}_{\mathrm{n}}\right)\right)$

Assign : $N \leftarrow\left(E_{1}^{\top}\left(\right.\right.$ or $\left.\left.\left(E_{1}\right)\right) \cup E_{2}\right)$.

Pass the bivector $B$ into the system $N$.

Let the resultant be $B N=B_{1} E_{1}^{\top}\left(\operatorname{or}\left(B_{1} E_{1}\right)\right) \cup B_{2} E_{2}=\left(y_{1}\right.$, $\left.y_{2}, \ldots, y_{m}\right)$ (or $\left(z_{1}, z_{2}, \ldots, z_{p}\right) \cup\left(x_{1}, x_{2}, \ldots, x_{n}\right)$.

Step 5 : Threshold and update this $\mathrm{BN}$ as:

$\mathrm{S}\left(\mathrm{y}_{\mathrm{j},},\right)=0$ if $\mathrm{y}_{\mathrm{j},} \leq 0 ; \quad 1 \leq \mathrm{j} \leq \mathrm{m}$

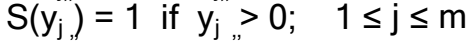

$\mathrm{S}\left(\mathrm{z}_{\mathrm{k},}\right)=0$ if $\mathrm{z}_{\mathrm{k},} \leq 0 ; \quad 1 \leq \mathrm{k} \leq \mathrm{p}$

$S\left(z_{k,}\right)=1$ if $z_{k}>0 ; \quad 1 \leq k \leq p$

$\mathrm{S}\left(\mathrm{x}_{\mathrm{i},}\right)=0$ if $\mathrm{x}_{\mathrm{i},} \leq 0 ; \quad 1 \leq \mathrm{i} \leq \mathrm{n}$

$S\left(x_{i}^{\prime \prime}\right)=1$ if $x_{i}^{\prime \prime}>0 ; \quad 1 \leq i \leq n$

That is if $\left(\mathrm{y}_{1}, \mathrm{y}_{2},{ }^{\prime}, \ldots, \mathrm{y}_{\mathrm{p}}{ }^{\prime \prime}\right)\left(\operatorname{or}\left(\mathrm{z}_{1}, \mathrm{z}_{2},{ }^{\prime}, ., \mathrm{z}_{\mathrm{m}}{ }^{\prime \prime}\right)\right) \cup\left(\mathrm{x}_{1}, \mathrm{x}_{2}, \ldots, \mathrm{x}_{\mathrm{n}}{ }^{\prime \prime}\right)$ $\longrightarrow\left(y_{1}, y_{2}, \ldots, y_{m}\right)$

(or $\left.\left(\mathrm{z}_{1}, \mathrm{z}_{2}, \ldots, \mathrm{z}_{\mathrm{p}}\right)\right) \cup\left(\mathrm{x}_{1}, \mathrm{x}_{2} \ldots \mathrm{x}_{\mathrm{n}}\right)$.

Moreover, keep the state $x_{j}$ as ON if it was in ON position in $\mathrm{B}_{2}$.

Then, the values of $x_{i}, y_{j}, z_{k}$ are either zero or 1 , for $1 \leq \mathrm{i}$ $\leq \mathrm{n} ; 1 \leq \mathrm{j} \leq \mathrm{m}$; and $1 \leq \mathrm{k} \leq \mathrm{p}$.

Step 6: Assign: $\left(\mathrm{A}_{1} \cup \mathrm{A}_{2}\right) \leftarrow\left(\mathrm{y}_{1}, \mathrm{y}_{2}, \ldots, \mathrm{y}_{\mathrm{p}}\right)$ (or

$\left.\left(\mathrm{z}_{1}, \mathrm{z}_{2}, \ldots, \mathrm{z}_{\mathrm{m}}\right)\right) \cup\left(\mathrm{x}_{1}, \mathrm{x}_{2} \ldots \mathrm{x}_{\mathrm{n}}\right)$.

Step 7: If $A_{1} \neq A_{1}^{\prime}$ and $A_{2} \neq A_{2}^{\prime}$ then

Assign: $A=\left(A_{1} \cup A_{2}\right) \leftarrow\left(A_{1} \cup A_{2}\right)$

Now this $A$ is the new input bivector.

Step 8: Repeat steps 2 through 7 till we get $A_{1}=A_{1}^{\prime}$ and $A_{2}=A_{2}$

Output: The pair of Limit Points.

3.5 The Advantages of new BAFCM bimodel

(i) The main advantage is that the method gives the occurrence of the resultant in pairs

by which stage by stage comparison is possible. (ii) When the data happens to be an upsupervised one the BAFCMs model becomes handy.

(iii) This bimodel also gives the hidden bipattern of the situation.

(iv) This bimodel gives equal status to each and every expert who gives his / her opinion.

(v) The only disadvantage is when we form the combined BAFCM bimodel and if in that

bimodel we have weightages 1 and -1 for some nodes we have the sum adding to

zero. Thus at all times the connection bimatrices may not be comfortable for addition.

(vi) Combined conflicting opinion tend to cancel out and assisted by the strong law of

large numbers. A consensus emerges as the sample opinion approximates the underlying population opinion. This problem will be easily overcome if in the BAFCM

bimodel the entries are only 0 and 1 .

Some properties of the BAFCM bimodel

(i) BAFCM are more applicable when the data in the first place is an unsupervised one.

BAFCMs work on the opinion of experts. BAFCMs bimodel the world as a collection of

biclasses and casual relation between biclasses.

(ii) BAFCMs are fuzzy signed directed bigraphs with feedback. The directed biedge $\quad e_{i j}=(\quad) \cup(\quad)$ from the bicausal concept $\left(X_{k} Y_{s}\right)$ (or $\left.Y_{s} X_{k}\right) \cup C_{i} C$ measures how much $\left(X_{k}\right.$ causes $Y_{s}\left(\right.$ or $Y_{s}$ causes $\left.X_{k}\right)$ ) $\cup\left(C_{i}\right.$ causes $\left.C_{j}\right)$. The time varying concept bifunction $\mathrm{Y}_{\mathrm{s}}(\mathrm{t}) \quad\left(\right.$ or $\left.\mathrm{X}_{\mathrm{s}}(\mathrm{t})\right) \cup \mathrm{C}_{\mathrm{i}}(\mathrm{t})$ measures the non negative occurrence of some fuzzy

event, perhaps the strength of a political sentiment, problems faced by persons with disability etc.

(iii) BAFCM model can be used to model several types of problems. In this paper we only

model the problems faced by the persons with disability and the problems faced by

the caretakers from the society relative to the persons with disability.

The merits of this new BAFCM bimodel

1. BAFCM bimodel allows experts to represent factual and evaluative concepts in an

interactive frame.

2. Experts can quickly draw BAFCM bimodel pictures or respond to the questionnaire.

3. Experts can consent or dissent to the local causal structure and perhaps the global

equilibrium.

4. BAFCM knowledge representation and inferencing structure reduces to simple bivector

bimatrix operations, favours integrated circuit implementation.

5. Yet an BAFCM equally encodes the experts' knowledge or ignorance, wisdom or prejudice. 
It is important to mention here that we make use of the BAFCM bimodel in a special way so that the comparison is possible at each stage.

Application of this bimodel to the problem of health hazards of rag pickers

The health problem of Rag pickers

The study of the health problems faced by the rag pickers happens to be an alarming one as they are unaware of the seriousness of the situation. When we say health problems this includes both physical and psychological. It is pertinent to keep in record that rag pickers do the job of cleaning the Chennai city as unpaid servants. However this job is done mostly by small children under the age of fifteen years. These children, when they should be in school are in streets doing the health risk job of picking rags to keep themselves free from starvation. Most of the rag pickers are runaway's from their home and are school dropouts. They have no permanent shelter. They sleep in railway stations, in trains, in bus stands, in corners of streets or near the waste disposed area itself. Because of this they face several health problems and suffer not only the seasonal attack of the diseases but suffer chronic diseases like malarial fever, typhoid cough incurable life threatening skin diseases and fever to which they succumb to. These children die in their prime life (that is even before they reach the age of 25-30 years).

We have collected data of 200 rag pickers from Chennai region. Since problems like, psychological problem, social problem leading to health hazards and other problems which cannot be measured as statistical data, we in this paper use our fuzzy bimodel to study their problems.

\section{Adaptation of the problem to BAFCM Bimodel}

Now using these linguistic questionnaires we have taken the following as the main attributes associated with the health hazards of the rag pickers and the influence of the public, parents and social circumstances. We have taken the following as the main attributes; it is not an hard and fast rule; one has to use only these. It is left to the choice of any researcher or includes or exclude any of the attributes.

We from the linguistic questionnaire see that the rag pickers take to several bad habits like dricking, ganza etc etc due to bad company. They say they take for sleep, as they cannot sleep in the nights in the roadside. Also they face several social problems like threat by police, for the public easily suspect them for any social problems. They are often taken into police custody for no fault of their. Further it is important to note that the wastes like medical waste, chemical waste which are very much injurious to their health are handled in bare hands at times they have open wounds in hands and limbs which are exposed to the poisonous waste, thereby making the wounds septic. Thus we see these rag pickers are exposed to several health hazards not only due to waste but also due to bad habits.
Vol. 5 No. 3 (Mar 2012) ISSN: 0974-6846
The linguistic questionnaire was transformed into a BAM as 7 main attributes of problems faced by the rag pickers and 8 attributes as the the cause of it which acts as catalyst. We list out brifly the main attributes and label them as $\left\{S_{1}, S_{2}, \ldots, S_{7}\right\}$ and $\left\{P_{1}, P_{2}, \ldots, P_{8}\right\}$ respectively as follows. Also through another expert linguistic questionnaire was transformed into a FCM as 7 main attributes of problems faced by the rag pickers and label them as $\left\{\mathrm{C}_{1}, \mathrm{C}_{2}, \ldots \ldots, \mathrm{C}_{7}\right\}$.

The Attributes are :

$\mathrm{S}_{1}$ - Quarrel at home / ill treatment.

$\mathrm{S}_{2}$ - School dropout.

$\mathrm{S}_{3}$ - Runaway from the family.

$\mathrm{S}_{4^{-}}$Rag Picking as an independent Profession

$\mathrm{S}_{5}$ - Take up to all bad habits due to several reasons like bad company,

for sleep, etc., so become drug addicts.

$\mathrm{S}_{6}$ - Poverty and seeks self respect.

$\mathrm{S}_{7}$ - No hygiene/ no knowledge about hygiene / about the hazardous waste

they deal with.

$P_{1}$ - Father a drunkard / No parents /mother dead/ parents in jail.

$\mathrm{P}_{2}$ - No motivation by teacher in school.

$\mathrm{P}_{3}$ - Enjoy independence /self support.

$\mathrm{P}_{4}$ - No place to sleep in night.

$\mathrm{P}_{5}$ - Problem given by Police.

$\mathrm{P}_{6}$ - Malaria / typhoid.

$\mathrm{P}_{7}$ - Scabies / hepatics / skin ailment due to rag picking.

$\mathrm{P}_{8}$ - Government and public has taken no steps to manage waste.

$\mathrm{C}_{1}$ : Left orphans due to parents/family members death.

$\mathrm{C}_{2}$ : School dropouts/no good school in the vicinity/ no money to pay school fees/

ill- treatment by the teachers.

$\mathrm{C}_{3}$ : Rag picking as a source of income and livelihood.

$\mathrm{C}_{4}$ : Bad company and Bad habits.

$\mathrm{C}_{5}$ : Parents in jail.

$\mathrm{C}_{6}$ : Quarrel at home with parents or family members.

$\mathrm{C}_{7}$ : - Poverty and umemployment.

As the data is an unsupervised one and involves lot of uncertainties we are justified in using BAFCM to analyze the problem the causes effect relation has been converted into a connection bimatrix $\mathrm{E}$.

\subsection{BAFCM model based on the expert's opinion}

Taking the neuronal field $F_{x}$ as the attributes connecting with the health problems and the field $F_{y}$ as the types of pollutions, the $7 \times 8$ matrix $E_{1}$ represents the forward synaptic projections form the neuronal field $F_{x}$ to the neuronal field $F_{y}$. The $8 \times 7$ matrix $E_{1}^{\top}$ represents the backward projection from $F_{y}$ to $F_{x}$. Now taking $S_{1}, S_{2}, \ldots$, $S_{7}$ along the rows and $P_{1}, P_{2}, \ldots, P_{8}$ along the columns we get the first component $E_{1}$ of synaptic connecting bimatrix $E$. Similarly, by taking $C_{1}, C_{2}, \ldots, C_{7}$ as rows as well as columns we get a $7 \times 7$ square matrix as the second component $E_{2}$ of the synaptic connecting bimatrix $E$. 
Now take this connection bimatrix of BAFCM bimodel which has both BAM and FCM components and take $A_{1}$ as the initial input bivector. In $A_{1}$, let a particular vector component, say $p_{1}$ and $s_{1}$, be kept on ON state and all other components are on OFF state and pass the state vector $A_{1}$ through the connection bimatrix $E$. To convert the resultant vector into a signal function, the values in the BAM component, which are greater than 0 are taken as 1 and less than or equal to 0 are taken as 0 in the signal function and in the FCM component, the state which is on ON position remains to be ON state and the values which are greater than or equal to one are made as ON state and all others as OFF state by giving values 1 and 0 respectively. Denote this process by the symbol $\longrightarrow$.

The first BAM component of the resulting vector is multiplied with $\mathrm{E}^{\top}$ and the second $\mathrm{FCM}$ component of the resulting vector is kept as it is and thresholding yields a new vector $A_{2}$. Using this new input bivector, we repeat the same procedure till a fixed point or a limit cycle is obtained. Next we choose the vector with its second vector components in both BAM and FCM components are on ON state and repeat the same to get another cycle. This process has been repeated for all the vectors separately. We observe the hidden pattern of some vectors found in all or many cases. Inference from this hidden pattern highlights the causes. We get the synaptic connecting bimatrix $E$ as follows:
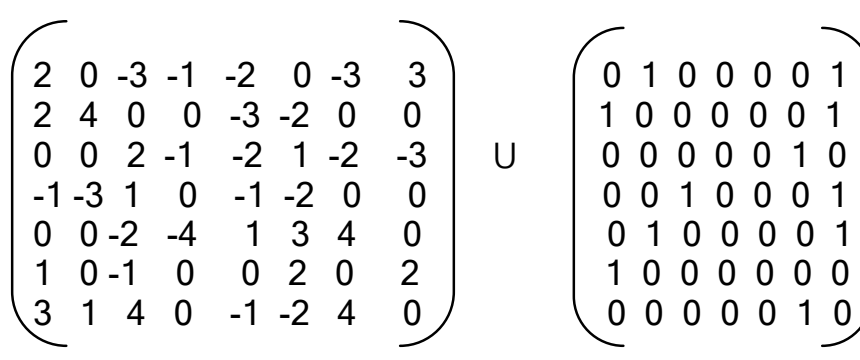

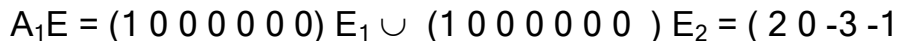

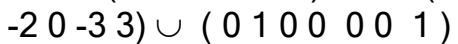

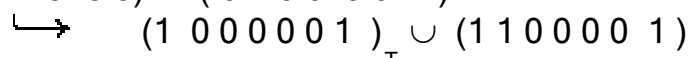

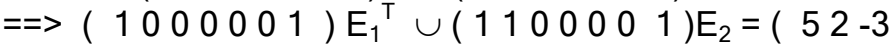

$$
\begin{aligned}
& -1013) \cup\left(\begin{array}{lllll}
11 & 0 & 0 & 0 & 11
\end{array}\right) \\
& \longrightarrow(1100011) \cup(1100011)=A_{2}
\end{aligned}
$$

Now $A_{1} \neq A_{2}$. Hence we proceed further to get the limit point as follows.

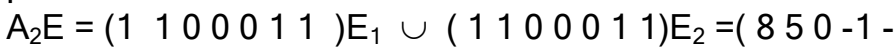
$6-215) \cup(2100012)$

\section{$\longrightarrow \quad(11000011) \cup(1100011)$}

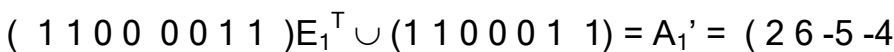

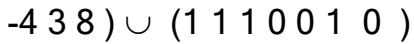

$\longrightarrow(1100011) \cup(1110010)=A_{3}$

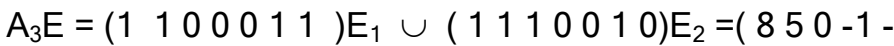
$6-215) \cup(2320030)$

\section{$\longrightarrow \quad(11000011) \cup(1110010)$}

Now $A_{2}=A_{3}$. Hence the limit point is: $\left(\begin{array}{llllll}1 & 1 & 0 & 0 & 0 & 11\end{array}\right)\left(\begin{array}{ll}11 \\ 1\end{array}\right.$

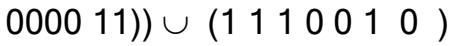

The set of limit points corresponding to different input vectors

\begin{tabular}{|c|c|c|}
\hline No & Input vector & Limit points \\
\hline 1 & (1000 000) & 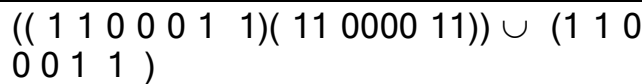 \\
\hline 2 & $(0100000)$ & 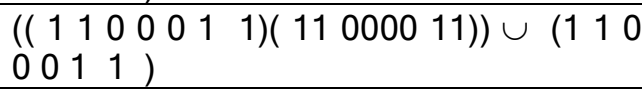 \\
\hline 3 & $(0010000)$ & 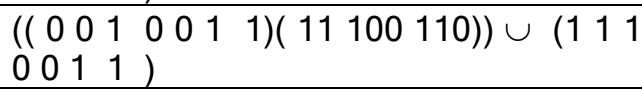 \\
\hline 4 & $(0001000)$ & 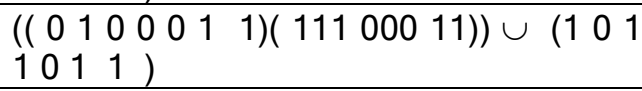 \\
\hline 5 & $(0000$ 100) & 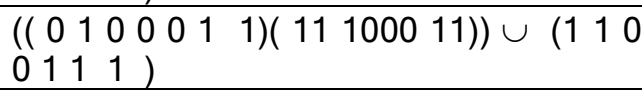 \\
\hline 6 & $(0000010)$ & 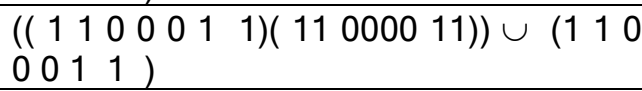 \\
\hline 7 & $(0000001)$ & 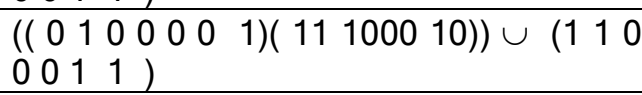 \\
\hline
\end{tabular}
are given in the following table.

\section{Analysis using $B=\mathrm{E}_{1} \cup \mathrm{E}_{2}$}

\section{Conclusion}

We analyzed the health problems of faced by the rag as ON state saying that left orphans due to parents/family members death is initializing attribute and all other attributes are left in the OFF state. The symbol $\longrightarrow$ stands for threshholding and it means that negative values are replaced by zeros, values greater than or equal to one or replaced by 1 and that component in FCM component which had been initially kept on ON state, will always be retained as $\mathrm{ON}$ state.

Let the initial vector $A_{1}$ in BAFCM bimodel be : (4 -3 -4 -2

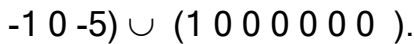

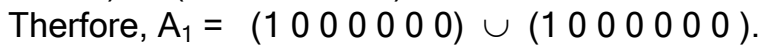
The effect of $A_{1}$ on the dynamical system $E$ is Research article COIndian Society for Education and Environment (iSee) the dynamical bisytem reveals that the attributes $P_{1}, P_{2}$, $\mathrm{P}_{7}, \mathrm{P}_{8}, \mathrm{~S}_{1}, \mathrm{~S}_{2}, \mathrm{~S}_{6}, \mathrm{~S}_{7}, \mathrm{C}_{1}, \mathrm{C}_{2}, \mathrm{C}_{6}$ and $\mathrm{C}_{7}$, are the main causes for their problems and health hazards. This means, father a drunkard or left orphans due to parents death or parents in jail, leads them to discontinue their studies. At some times due to no motivation by teacher in school or ill- treatment by the teachers or no money to pay school fees, the quarrel at home with parents or family members, they become school dropout. They are suffering from poverty and umemployment. But they 
seeks self respect. They have seen that the Government and public has taken no steps to manage waste. Also, they do not have any knowledge about hygiene and about the hazardous of the waste if they deal with it. This leads them to enter in to the profession of Rag picking. Finally they suffer with many diseasies such as scabies, hepatics and skin ailment due to rag picking.

\section{Suggestions}

1. Steps must be taken to retain children in schools.

2. Government should take legal steps to eradicate child labour. Implementation of existing laws must be made stringent.

3. Steps must be given to provide them free adult education in the nights after hours of works. (It is pertinent to mention here that certain groups are supporting a few of the rag pickers in the city).

4. The rag pickers must be approached by some social workers (who work on non profit basis) to advice these rag pickers about the health problems and risks they face by rag picking.

5. Public must be provided with civic sense not to dump hazardous waste were rag pickers do the rag picking.

6. Government should take strong step both to prevent the rag pickers around the hospital zone and also the hospital authorities not to dumb the dangerous and hazardous wastes which are reachable by the rag picker.

7. Government must provide them freely good gloves and shoes while going for rag picking.

\section{References}

1. Balasangu $\mathrm{K}$, Thirusangu $\mathrm{K}$ and Rajkumar Dare $\mathrm{V}$ (2007) A fuzzy approach on the analysis of health hazards faced by the agriculture labourers due to chemical pollutions. Proc. Indian Conf. on Intelligent Systems, Allied Publ.. pp: 123-128.

2. Balasangu $\mathrm{K}$, Thirusangu $\mathrm{K}$ and Rajkumar Dare $\mathrm{V}$ (2009) On the health hazards of the rice cultivators using IFRM model. IJAMCCBM. 1 (1), 1-10.

3. Balasangu $\mathrm{K}$, Thirusangu $\mathrm{K}$ and Rajkumar Dare $\mathrm{V}$ (2011) IFAM model approach on the impact of pesticides on agricultural labourers. Indian J.Sci. Technol. 4 (2), 151-154.

4. Klir GJ and Folger TA (1998) Fuzzy sets, uncertainty, and information. Prentice Hall, New Jersey.

5. Kosko B (1988) Bidirectional associative memories. IEEE Transact. Systems, Man \& Cybernetics, SMC18, 49-60.

6. Kosko B (2001) Neural networks and fuzzy systems. Prentice Hall, India.

7. Pathinathan $T$, Thirusangu $K$ and Mary John (2005) On causes for school dropouts- A fuzzy approach. Acta Ciencia Indica, XXXI M, (4) 1279-1299.

8. Praveen Prakash A (2010) Analysis of the problems faced by the rural disadvantaged persons with disabilities - Using new fuzzy bimodels, Ph.D., Thesis, University of Madras.
Vol. 5 No. 3 (Mar 2012)

ISSN: 0974- 6846

9. Zadeh LA (1965) Fuzzy sets. Information Control. (8) 338-353. 